\title{
Dissociative amnesia - a multiple case study
}

\author{
Angelica Staniloiu ${ }^{1,2,3}$ Hans J. Markowitsch, ${ }^{2}$ Andreas Kordon ${ }^{1,4}$ \\ ${ }^{1}$ Psychiatry, Oberbergklinik Hornberg, Germany, ${ }^{2}$ Psychology, University of Bielefeld, \\ Germany, ${ }^{3}$ Psychology, University of Bucharest, Romania, ${ }^{4}$ Psychiatry, University of \\ Freiburg, Germany
}

\section{Introduction and Objectives}

Autobiographical amnesia is found in patients with focal or diffuse brain damage ("organic amnesia"), but also in patients without overt brain damage (as measured with conventional brain imaging). This last condition is usually named 'dissociative amnesia'. Related terms are 'psychogenic amnesia', 'functional amnesia', or the 'mnestic block syndrome' (Staniloiu and Markowitsch, 2014; Markowitsch and Staniloiu, 2016).

Most dissociative amnesias are retrograde (Fig. 1) and affect processing within the episodic-autobiographical memory system. Processing within other memory systems is usually preserved (cf. Fig. 2).

27 patients with psychogenically caused autobiographical amnesia are described to establish variables which are central for the occurrence of dissociative amnesia.

Methods: Psychiatric and psychological interviews, neuropsychological tests, and brain imaging methods were applied.

Results: Main features of the cases are demonstrated in Table 1.

Conclusions: Autobiographical amnesia of psychogenic origin is a heterogenous condition. It varies with respect to precipitants, clinical manifestations and course.
References: Markowitsch, H. J. \& Staniloiu, A. (2016). Functional (dissociative) retrograde amnesia. In M. Hallett, J. Stone \& A. Carson (Eds.) Handbook of clinical neurology (3rd series): Functional neurological disorders (pp. 419-445). Amsterdam: Elsevier; Staniloiu, A. \& Markowitsch, H. J. (2014). Dissociative amnesia. Lancet Psychiatry, 1, 226-241.

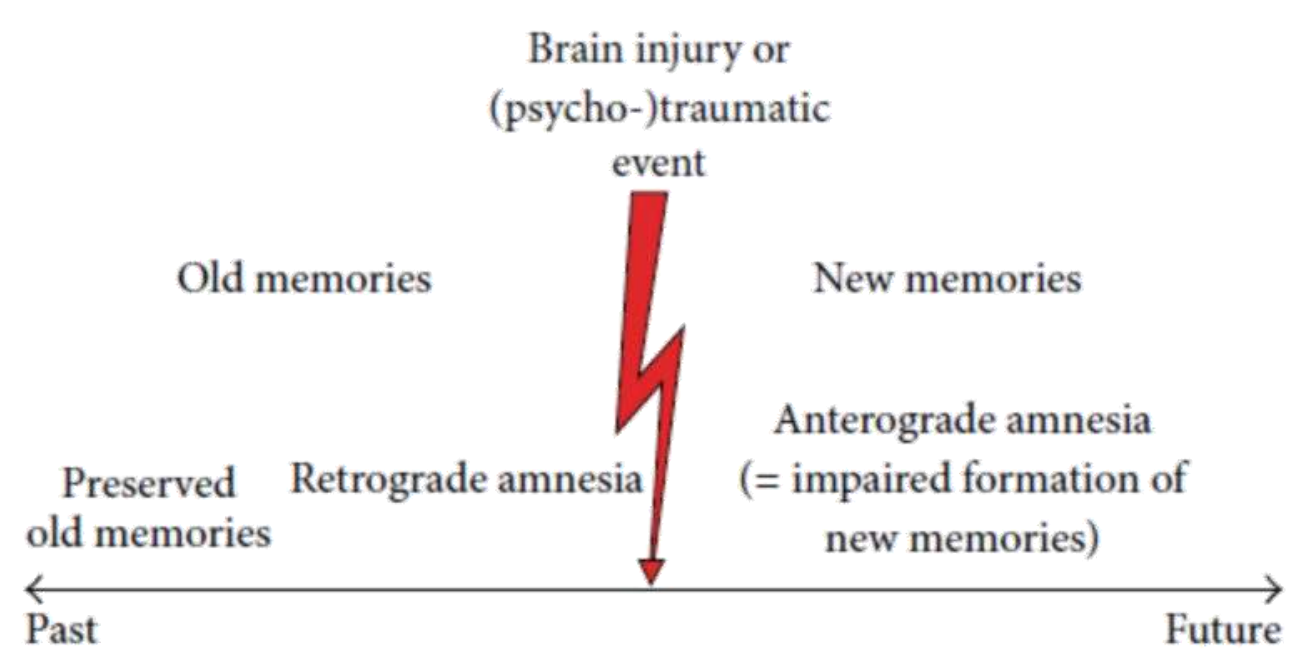

Fig. 1. Relations between stress or brain damage related anterograde and retrograde amnesia.

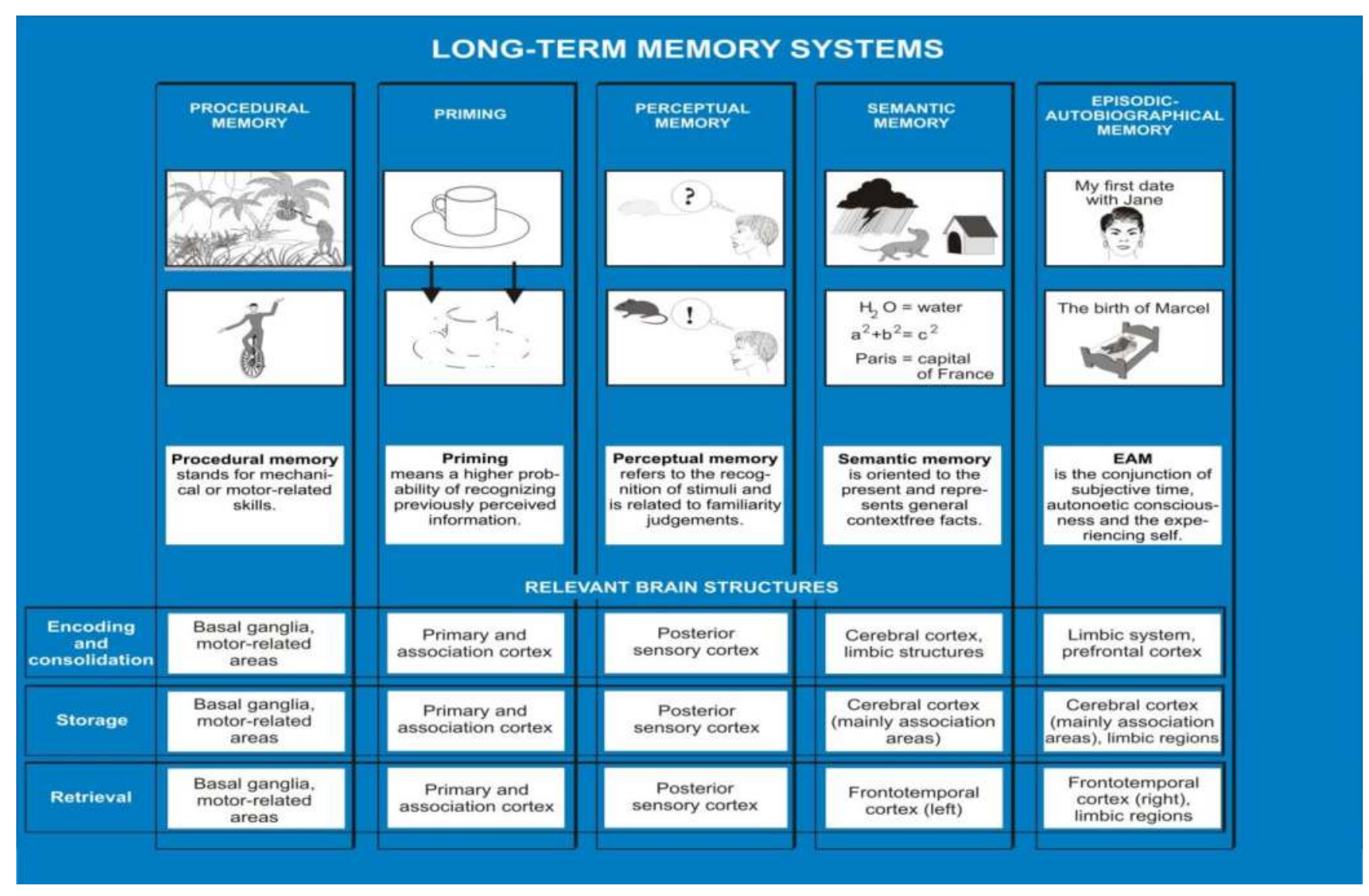

Fig. 2. The five long-term memory systems.

\begin{tabular}{|c|c|c|c|c|c|c|c|c|c|c|c|c|c|c|c|c|c|c|c|c|c|c|c|c|c|c|}
\hline Patiens & A & B & c & $\mathrm{D}$ & $\bar{E}$ & $F$ & $\bar{G}$ & $\mathrm{H}$ & 1 & 1 & K & $\bar{L}$ & $\mathrm{M}$ & $\mathrm{N}$ & o & $P$ & $\bar{a}$ & $R$ & s & $T$ & u & $\mathrm{v}$ & $\mathrm{w}$ & $\bar{x}$ & $z$ & $\mathrm{ZA}$ \\
\hline $\begin{array}{l}\text { Ase. } \\
\text { Gendef }\end{array}$ & Nale & $\begin{array}{l}26 \\
\text { wale }\end{array}$ & Male & $\frac{52}{\text { mile }}$ & $\begin{array}{ll}30 \\
\text { semant }\end{array}$ & $\frac{21}{\text { male }}$ & 22 & Male & $\begin{array}{ll}44 \\
\text { Male }\end{array}$ & 4 & Wale & $\begin{array}{l}\text { mile } \\
\text { Mile }\end{array}$ & 15 renat & $\frac{11}{113 e}$ & fement & $\frac{35}{\text { fement }}$ & 22 & $\frac{s^{50}}{{ }_{\text {male }}}$ & $\frac{26}{2 \text { mar }}$ & 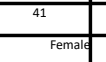 & $\frac{52}{6 \text { mate }}$ & 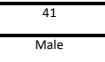 & $\begin{array}{l}31 \\
\text { Male }\end{array}$ & $\begin{array}{ll}52 \\
\text { Nale }\end{array}$ & $C_{\text {mae }}^{37}$ & Male \\
\hline Education & 111verar & 111 vear & 11 verat & 16 veat & 13 seart & 111 yea & Tyeass & 12 2reart & 8 seat & 6ryas & 8 searest & 13 veras: & 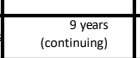 & 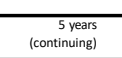 & 17veras & 8 sear & 13 eara & 11 veas: & 11 11ears: & 11 veraf & 8 seat & 8 year & 8 vear & ${ }_{11 \text { year: }}$ & 13 seat & \\
\hline $\begin{array}{l}\text { Family } \\
\text { background }\end{array}$ & partree & No partine & merare & 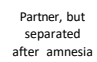 & Partine & Partne & No postane & No pastren & Marief & Marrie & Pastent & marrica & Not sponicase & Not applicab & No partane & Marries & No parten & No parane & Noporanes & Marice & Marrie & Marriea & Marrica & marrest & Partane & Noc sonen \\
\hline Branıimag & ме1 & & ме1 & 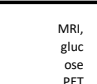 & ме1 & & - & мख1 & व & ме1 & & 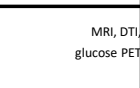 & . & - & . & - & ст & - & мен & ст & ст & мев & мев & . & . & \\
\hline $\begin{array}{c}\text { Psychilaticic } \\
\text { historyatic } \\
\text { somant } \\
\text { complaints }\end{array}$ & 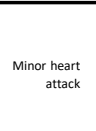 & uninow & uninowe & untaon & 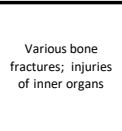 & 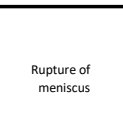 & untrowe & Untrowe & strionof & 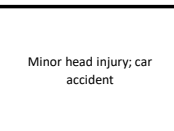 & untroun & 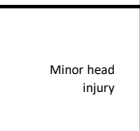 & 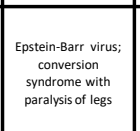 & uninow & unknow & 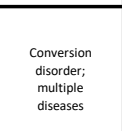 & 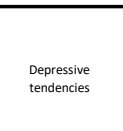 & unkrowe & unnow & Unther & Timonet & 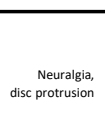 & Unk & 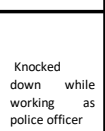 & untroment & \\
\hline $\begin{array}{l}\text { Stressful events } \\
\text { /circumstances }\end{array}$ & 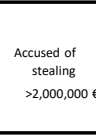 & 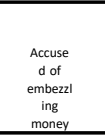 & craracioner & 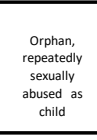 & crarcciden & 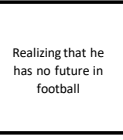 & netreserf & vanowem - & 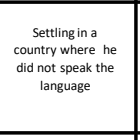 & 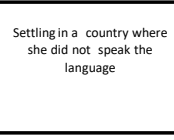 & 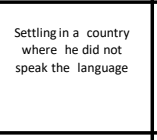 & 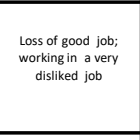 & 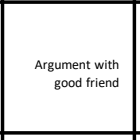 & 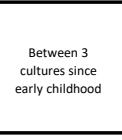 & vinower & 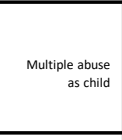 & 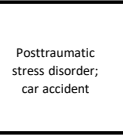 & untroun & Untroon & unan & untrowe & & Unkeno & 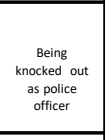 & 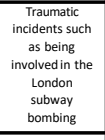 & 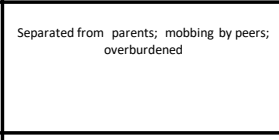 \\
\hline & 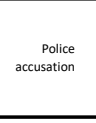 & 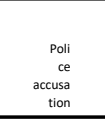 & 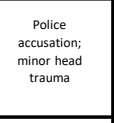 & 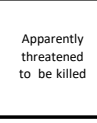 & 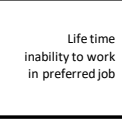 & 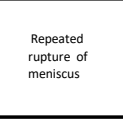 & Untrour & 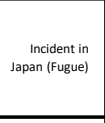 & $\begin{array}{c}\text { Mnor hesed } \\
\text { injury }\end{array}$ & 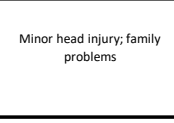 & froven & 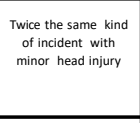 & 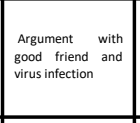 & 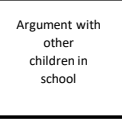 & 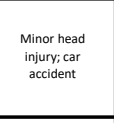 & surear & 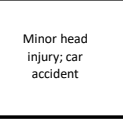 & frovere & unnow & 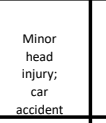 & 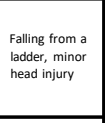 & caracciden & 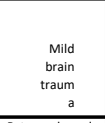 & 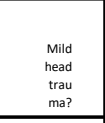 & 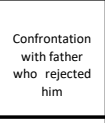 & Mober \\
\hline & Retergasad & Retrogarad & Rectogesat & 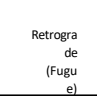 & Rerergast & Retrogat & 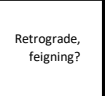 & Retrot & Retroger & Rereseract & Retrogrased & & Reart & Reregesa & nearc & Rerergased & Retrogerad & & 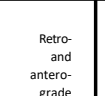 & Reroge & Rerergased & Rerergased & 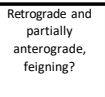 & Retro & Rerergased & \\
\hline $\begin{array}{l}\text { hesictime } \\
\text { period }\end{array}$ & Trosulite & 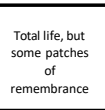 & Toallith & Trostill & Trablite & Toabliti & Toallitit & Trating & rosilnt & Toablitit & rosalnt & & Totallites & Troblit & Toally & bastat a very & 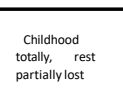 & Trostint & Toabl & rosalut & 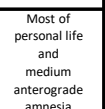 & Trablint & Tobalitut & Toos & robally & ot \\
\hline Intelligence & 9 & $\phi$ & 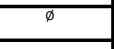 & 910 & - & 8 & 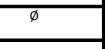 & $\bar{s}$ & ofor & $\downarrow$ & & & 0.000 & 910 & 6 & 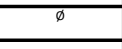 & ${ }^{\circ}$ & & 8 & $\phi$ & 9 & $\bar{\sigma}$ & 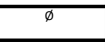 & 8 & 80101 & \\
\hline $\begin{array}{l}\text { Executuve } \\
\text { functions }\end{array}$ & . & 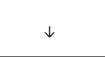 & $\downarrow$ & $\circ$ & $\downarrow$ & . & $\downarrow$ & . & $\downarrow$ & 。 & 0.001 & orou & $\uparrow$ & . & $\uparrow$ & $\downarrow$ & $\varnothing$ & $\varnothing$ & $\downarrow$ & $\varnothing$ & . & $\phi$ & $\downarrow$ & $\downarrow$ & $\downarrow$ & $\varnothing$ \\
\hline $\begin{array}{c}\text { Antention \& } \\
\text { concentration }\end{array}$ & $\downarrow$ & $\phi$ & $\downarrow$ & $\circ$ & $\downarrow$ & $\phi$ to & $\downarrow$ & $\varnothing$ & $\downarrow$ & $\downarrow$ & 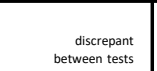 & $\downarrow$ & $\uparrow$ & 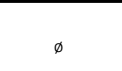 & $\uparrow$ & $\downarrow$ & $\phi$ to & 9001 & $\downarrow$ & $\varnothing$ & $\downarrow$ & $\circ$ & $\downarrow$ & $\downarrow$ & $\downarrow$ & 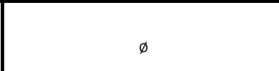 \\
\hline & Blune & 。 & Bune & $\circ$ & 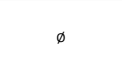 & $\circ$ & sung & Buone & Bune & Bune & 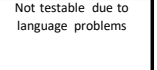 & sune & 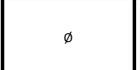 & 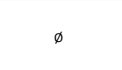 & $\circ$ & $\circ$ & 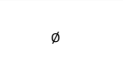 & sun & 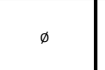 & 9007 & $\circ$ & $\circ$ & Bunt & $\circ$ & sunt & Bunt \\
\hline
\end{tabular}

